\title{
Ein paar Worte zum Abschnitt E (= Etymologie) des LfgrE
}

\author{
MICHAEL MEIER-BRÜGGER
}

1) Das ideale Lemma eines LfgrE-Artikels besteht aus den folgenden Informationsteilen:

- der Frequenzangabe $\left[\mathrm{x}^{\mathrm{i}}, \mathrm{x}^{\mathrm{o}}, \mathrm{x}^{\mathrm{H}}, \mathrm{x}^{\mathrm{h}}, \mathrm{x}^{\mathrm{e}}\right]$ und den Teilen E, F, M, $\Sigma \mathrm{X}, \mathrm{L}$, B, D, G.

- $\quad \mathrm{E}=$ "genet. Abdruck" = Nennung von Wortverband mit Ableitungen und Vorgeschichte,

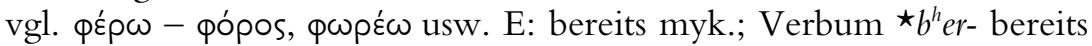
im Lexikon der urindogermanischen Grundsprache vorhanden $=$ ererbt

2) Ein Beispiel, um die Problematik von E zu verdeutlichen:

2a) Kriegsziel der Griechen vor Troia:

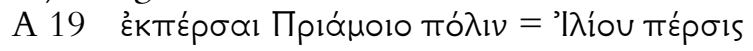

2b) Was bedeutet $\pi \dot{\varepsilon} \rho \theta \omega$ konkret? Welcher Aspekt ist herausgegriffen?

- wie dt. "erobern" i.e. "der Obere bleiben / Oberhand behalten"?

- wie dt. "einnehmen"

- wie dt. "plündern" i.e. "Plunder (Hausrat)" wegnehmen?

- wie dt. "zerstören" i.e. "zerstreuen / vernichten"?

2c) Antike Zerstörungen waren radikal: Städte wurden dem Erdboden gleichgemacht.

- vgl. Perserschutt auf der Akropolis

- vgl. die Zerstörung Milets durch die Perser (archaischer Schutt am Kalabaktepe)

2d) Neuer Vorschlag:

- die bis jetzt vorgelegten Analysen überzeugen nicht, auch nicht M. Janda, Eleusis, Innsbruck 2010, p. 230-241 $\left(<\star / b^{h} e r-d^{h} h_{1-} /\right.$ "das Davontragen")

- trotz der expliziten Ablehnung durch Frisk: $\pi \varepsilon \dot{p} \theta \omega$ ist gleich gebildet wie lat. perdere

- lat. per- + -dere aktiv "zu Grunde richten", "destroy", <*"hinüber machen" 
- pass. dazu per- + -ire "zu Grunde gehen", "disappear", < "hinüber gehen"

- per-dere wie con-dere "gründen"

- -dere zu -da- $<\star d^{h} h_{1}$ - i.S.v. "machen"

- $\quad \mathrm{zu}-d a-$ altes Perfekt $f \bar{e}-c_{-}<\star^{h} d^{h} h_{1^{-}}$, woraus neu lat. facere

- im Griech. *per $+d^{h} h_{1^{-}}>\pi \varepsilon \dot{\varepsilon}-\theta \omega /$ हैm $\pi \propto \theta \circ v$ "zu Grunde richten"

- vgl. है $\mu \alpha \theta \circ v(\mu \alpha v \theta \alpha \dot{\alpha} v \omega)<\star_{m}^{\circ} n s-d^{h} h_{1-}$ "in den Geist legen"

- dagegen ein Präsens wie $\pi \lambda \eta \dot{\theta} \omega \omega$ "bin voll" in Umkreis des pass. Aor.

2e) Quintessenz zum Abschnitt E

- Etymologien lassen sich nicht systematisch finden

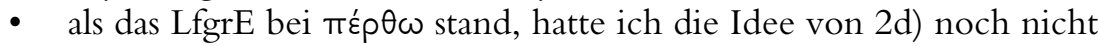

- wenn neue Etymologien auftauchen, brauchen sie Platz für eine überzeugende Argumentation und E gibt dafür den Raum nicht her

- Andeutungen allein sind zu vage, nur bereits vorliegende überzeugende Publikationen sind zitierfähig

3) Wie war der Umgang des LfgrE mit E?

3a) Phase I im Band I Alpha

- sehr ausführlich

3b) Phase II mit Beginn Beta

- radikale Kürzung ohne viele Verweise

3c) Phase III ab Lief. 13

- Neue Handbücher verfügbar: Chantraine, Dict.; Mayrhofer, EWAia, LIV $^{2}$

- Hinweis darauf genügt i.d.R.

- nur ganz selten spezieller Aufsatz genannt

4) Meine schönsten Erlebnisse beim Bearbeiten von Abschnitt $E$

4a) Die Diskussionen zu E beim Entstehen der Artikel:

- Bei der E-Arbeit immer wieder die folgenden Informationen zu finden: "unklar" (Frisk), "n'est pas claire" (Chantraine)

- Diskussionen mit den Mitarbeiter sehr fruchtbar

- Erkenntnisse: "vieles ist nur vorhistorisch, aber nicht homerisch nachweisbar", "Wörter haben meist bereits eine lange Vorgeschichte hinter sich", vgl. ícpós

- richtige Idee für eine Lösung kommt oft unerwartet, läßt sich nicht erzwingen

4b) Mein schönster Fund ist die Etymologie von żá $\phi \theta \eta 2^{\mathrm{i}}$

- publiziert in Münchener Studien zur Sprachwissenschaft 50, 1989, 9196

- hatte u.a. "im Hinterkopf gespeichert" żá $\phi \theta \eta=$ "unklar" 


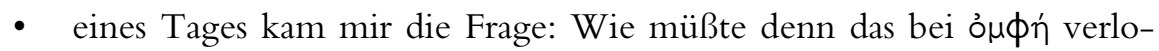
rene Verbum lauten?

- ̇uфń "Stimme" bekanntlich $<\star$ homp $p^{h} \bar{a}-<\star_{\text {song }} g^{w h} \bar{a}-$ "Gesang"

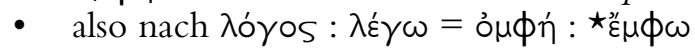

- AHA: źá $\phi \theta \eta$ ist der Aorist dazu!!!

- daher meine etymologische Lösung: $\dot{\varepsilon}-\alpha ́ \alpha-\theta \eta / e-a p^{h}-t^{h} \bar{e} /<\star / e-s^{0} n g^{w h}-$ $d^{h} \bar{e} /$

- $\quad \star /-s^{\circ} n g^{w h}-/ \mathrm{zu} \star / s e n g^{w h}-/$ "singen", belegt in ỏ $\mu \phi \eta ́$

- Lautlich: $\star /-s^{\circ} n g^{w h}-/$ wird frühgriech. zu /-hak $k^{w h}-/$ bzw. /-hap ${ }^{h}-/$ bzw. / $-a p^{h}-1$

- (im Frühgriechischen werden die Labiovelare beseitigt und werden vor dunklen Vokalen und Konsonanten zu Labialen; das neu entstandene $/-h-/$ ist wegen des folgenden $/-k^{w h}-/$ dissimiliert)

- Inhaltlich: źá $\phi \theta \eta=($ der Schild, der vom Speer getroffen zu Boden fällt) "wurde zum Singen/Klingen gebracht" 
Bereitgestellt von | SUB Göttingen Angemeldet

Heruntergeladen am | 27.10.14 12:34 